GANIT J. Bangladesh Math. Soc. (ISSN 1606-3694) 37 (2017) 111-119

\title{
STABILITY ANALYSIS OF A NONLINEAR SYSTEM WITH DIFFERENT GROWTH FUNCTIONS
}

\author{
Md. Kamrujjaman ${ }^{1}$ and Ashrafi Meher Niger \\ Department of Mathematics, University of Dhaka, Dhaka-1000, Bangladesh \\ ${ }^{1}$ Corresponding author: kamrujjaman@du.ac.bd
}

Received 16.05.2017 Accepted 26.10.2017

\begin{abstract}
A competitive mathematical model for the growth of two species is considered in this study. The main goal of the present study is to investigate the roles of two different growth functions: the logistic growth and the food limited growth. We established the main results that determine the asymptotic stability of semi-trivial as well as the coexistence solutions. If higher carrying capacity is embodied for the population following logistic growth then competitive exclusion of a food limited population is imminent and vice versa.
\end{abstract}

Keywords: Equilibria; competition; phase portrait; coexistence

\section{Introduction}

Extensive level of competition among the species is practically prevalent in every level of natural world. Mathematical models are developed and analyzed which provide information on the growth rates of two species under low (Malthusian growth) and high (logistic growth) densities, when they are only affected by their own populations, which is known as intraspecific competition [1]. Extended models are studied to determine how these species interact when competing for the limited resources, which is called interspecific competition [6]. Interspecific competition is detrimental to both species per capita growth rates. In the literature, the well developed and most popular growth laws were established in [2, 3, 7, 9]. There are different biological considerations to two species competition models using different growth functions of $[2,3,7,9]$. In this paper, a competition model is introduced by considering the concept of critical population density that follows different growth laws. Therefore, the goal of the present study is to develop a basic competition model based on two different species either in interspecific or intraspecific competition with two different growth rates.

The paper is organized as follows. In section 2, we describe the auxiliary result to analyze the model and a brief description of the reaction terms for species $u$ and $w$, and these will be used in the rest of the paper. Equilibrium analysis of semi-trivial steady states and coexistence solutions are investigated in section 3 by linearizing our considered problem. 
The key ingredients of Section 4 are to consider different carrying capacities for numerical simulations supporting the theoretical results and this characterization is referred to as the crowdiness effect. In this case, we show that the population following the food limited growth goes to extinction if higher crowdiness tolerance is incorporated with the logistic population for particular $\beta$. Finally, Section 5 presents summary of the results.

\section{Method of Analysis}

Taking into account of two different species, we consider the following system of nonlinear differential equations

$$
\left\{\begin{array}{l}
\frac{d u}{d t}=r_{1} u(t)\left(1-\frac{u(t)+w(t)}{K_{1}}\right) \\
\frac{d w}{d t}=r_{2} w(t)\left(\frac{1-\frac{u(t)+w(t)}{K_{2}}}{1+\beta \frac{u(t)+w(t)}{K_{2}}}\right)
\end{array}\right.
$$

where, $u(t)$ and $w(t)$ represent the density of the populations at time $t$, and $K_{1}, K_{2}$ are the carrying capacities of the respective species $u$ and $w$, respectively. Similarly, $r_{1}, r_{2}$ are the two intrinsic growth rates of $u$ and $w$, respectively, and $\beta$ is a positive constant. There are various methods to analyze the stability of nonlinear systems. Some popular methods of stability of nonlinear systems are Lyapunov satbility criteria $[8,10]$ and LaSalle's invariance principle $[4,5]$.

For mathematical analysis of the model (1), we consider the Hartman-Grobman theorem as described below:

Theorem 1. [6] (Hartman-Grobman) If the linearization matrix has no zero or pure imaginary eigenvalues then the phase portrait for the system near the equilibria $(u, v)$ can be obtained from the phase portrait of the linear system via a continuous change of coordinates.

Remark 1. In particular, this means that if the matrix has no zero or pure imaginary eigenvalues, then the stability properties of the equilibria $(u, v)$ of the system are the same as those of the equilibrium 0 of the linear system.

It is rare that an analytic formula for the solution of (1) can be constructed, so we usually must construct a numerical solution or study the qualitative behavior of the solution. A qualitative analysis proves extremely helpful for constructing numerical solutions. In either case, we find it convenient to study the solutions in the phase plane $(u, w)$.

For simplicity, let us introduce the following two functions 


$$
\begin{aligned}
& f(u, w)=r_{1} u(t)\left(1-\frac{u(t)+w(t)}{K_{1}}\right) \\
& h(u, w)=r_{2} w(t)\left(\frac{1-\frac{u(t)+w(t)}{K_{2}}}{1+\beta \frac{u(t)+w(t)}{K_{2}}}\right) .
\end{aligned}
$$

and rewrite the system (1) as follows:

$$
\begin{aligned}
& \frac{d u}{d t}=f(u, w) \\
& \frac{d w}{d t}=h(u, w)
\end{aligned}
$$

Any solution

$$
v(t)=\left(\begin{array}{l}
u(t) \\
w(t)
\end{array}\right)
$$

of the system (2) can be thought of as a parametrized curve which we refer to as integral curves of the system. We can use the fact that the vector $(d u / d t, d w / d t)$ is tangent to the solution curve defined by $(u(t), w(t))$. A graph of a family of solutions is called a phase portrait and can be generated from the direction field or numerically. A disadvantages of both the direction filed or numerical approach is that the parameters in (2) need to be specified.

Since equilibria of a system occur at points where the coordinates have derivative zero simultaneously. The equilibrium, steady state or critical points are constant solutions, $u(t)=u^{*}$ and $w(t)=w^{*}$, which satisfy the nonlinear system of equations $f(u, w)=0$ and simultaneously $h(u, w)=$ 0 . The equilibria of the system (2) are $\left(u^{*}, w^{*}\right)=(0,0),\left(K_{1}, 0\right),\left(0, K_{2}\right)$. Later sections for test out of the stability analysis at the equilibira.

\section{Stability Analysis at the Equilibria}

In this section, first we have linearized the system for further analysis. Rearrange the functions $f(u$, $w)$ and $h(u, w)$ as follows

$$
\begin{aligned}
& f(u, w)=\frac{r_{1}}{K_{1}}\left(u K_{1}-u^{2}-u w\right) \\
& h(u, w)=r_{2}\left(\frac{K_{2} w-u w-w^{2}}{K_{2}+\beta u+\beta w}\right)
\end{aligned}
$$

To find the linearization matrices at the equilibria, we first calculate 


$$
\begin{aligned}
& f_{u}=\frac{r_{1}}{K_{1}}\left(K_{1}-2 u-w\right) \\
& f_{w}=\frac{r_{1}}{K_{1}} u \\
& h_{u}=-\frac{r_{2} K_{2} w(1+\beta)}{\left(K_{2}+\beta u+\beta w\right)^{2}} \\
& h_{w}=\frac{r_{2}\left(K_{2}^{2}-K_{2} u-2 K_{2} w+\beta u K_{2}-2 \beta u w-\beta u^{2}-\beta w^{2}\right)}{\left(K_{2}+\beta u+\beta w\right)^{2}}
\end{aligned}
$$

Substituting the coordinates of the equilibria into these formulae, we obtain the linearization matrices.

Equilibrium (0, 0): At the equlibrium point $(0,0)$, four partial derivatives are

$$
f_{u}(0,0)=r_{1} ; f_{w}(0,0)=0 ; h_{u}(0,0)=0 \text { and } h_{w}(0,0)=r_{2}
$$

Then the linear system

$$
\begin{aligned}
& \frac{d u}{d t}=r_{1} u \\
& \frac{d w}{d t}=r_{2} w
\end{aligned}
$$

and the linearization matrix at $(0,0)$ is

$$
M_{(0,0)}=\left(\begin{array}{cc}
r_{1} & 0 \\
0 & r_{2}
\end{array}\right)
$$

corresponding eigenvalues of $M_{(0,0)}$ are $\lambda_{1}=r_{1}>0, \lambda_{2}=r_{2}>0$. Put $r_{1}=10$ and $r_{2}=5$ which gives $\lambda_{1}=10, \lambda_{2}=5$ and the eigenvectors

$$
\left(\begin{array}{l}
1 \\
0
\end{array}\right) \text { and }\left(\begin{array}{l}
0 \\
1
\end{array}\right)
$$

respectively.

Both eigenvalues of the linearization matrix $M_{(0,0)}$ are real and positive which concludes that the equilibrium point $(0,0)$ is a repeller. Biologically, when both of the species are present in the same ecological niche they will repel each other and leave the sub-domain of the habitat until unless other factors are considered. At this critical point the system is always unstable, see Fig. 1. 


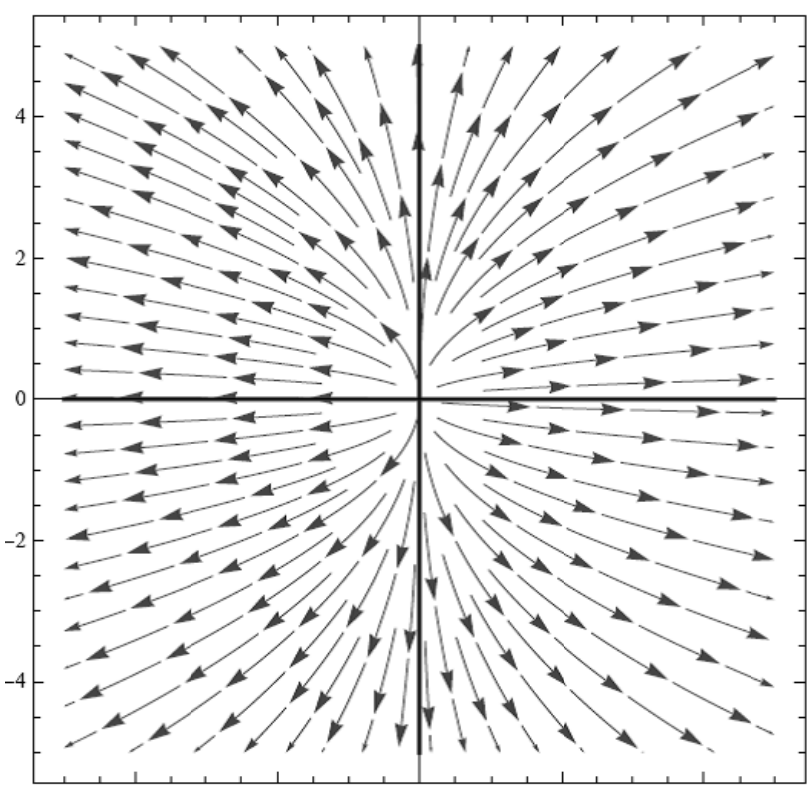

Figure 1. Eigensystem and phase portrait of the linear system (4) near $(0,0)$.

Equilibrium $\left(\mathbf{0}, \boldsymbol{K}_{\mathbf{2}}\right)$ : We can find the linear system after calculating the following terms

$$
f_{u}\left(0, K_{2}\right)=\frac{r_{1}\left(K_{1}-K_{2}\right)}{K_{1}} ; f_{w}\left(0, K_{2}\right)=0 ; h_{u}\left(0, K_{2}\right)=-\frac{r_{2}}{(1+\beta)}
$$

and $h_{w}\left(0, K_{2}\right)=-r_{2} /(1+\beta)$

Then the linear system will be

$$
\begin{gathered}
\frac{d u}{d t}=\frac{r_{1}\left(K_{1}-K_{2}\right)}{K_{1}} u \\
\frac{d w}{d t}=-\frac{r_{2}}{1+\beta}\left(u+w-K_{2}\right)
\end{gathered}
$$

and the linearization matrix at $\left(0, K_{2}\right)$ is

$$
M_{\left(0, K_{2}\right)}=\left(\begin{array}{lc}
r_{2}\left(K_{1}-K_{2}\right) / K_{1} & 0 \\
-r_{2} /(1+\beta) & -r_{2} /(1+\beta)
\end{array}\right)
$$

Eigenvalues of the matrix $M_{\left(0, K_{2}\right)}$ are $\lambda_{1}=-r_{2} /(1+\beta)<0$ and $\lambda_{2}=r_{1}\left(K_{1}-K_{2}\right) / K_{1}$, where $\lambda_{2}$ is positive when $K_{1}>K_{2}$ and negative for $K_{1}<K_{2}$.

As an example, put $\beta=2, r_{1}=3, r_{2}=5$ and for $K_{1}=4, K_{2}=3$, we have $\lambda_{1}=-5 / 3, \lambda_{2}=3 / 4$ and the corresponding eigenvectors

$$
\left(\begin{array}{l}
0 \\
1
\end{array}\right) \text { and }\left(\begin{array}{c}
-29 / 20 \\
1
\end{array}\right) \text {, }
$$


respectively. Two eigenvalues of the matrix $M_{\left(0, K_{2}\right)}$ are both real and have opposite signs. Thus the equilibrium $\left(0, K_{2}\right)$ is an unstable saddle point as shown in figure 2 (left). In oral habitat, if the species $u$ enjoys higher level of natural resources then it will out-compete as the species $w$ following the food limited growth.

Similarly, for $K_{1}=3, K_{2}=4$ with same $\beta, r_{1}$ and $r_{2}$ as above, $\lambda_{1}=-5 / 3, \lambda_{2}=-1$ with respective eigenvectors

$$
\left(\begin{array}{l}
0 \\
1
\end{array}\right) \text { and }\left(\begin{array}{c}
-2 / 5 \\
1
\end{array}\right)
$$

Since the eigenvalues $\lambda_{1}$ and $\lambda_{2}$ both are negative, then the critical point $\left(0, K_{2}\right)$ is an asymptotically stable node shown in figure 2 (right). Therefore, if the second species has more accessibility to the resources then the first population goes to extinction.
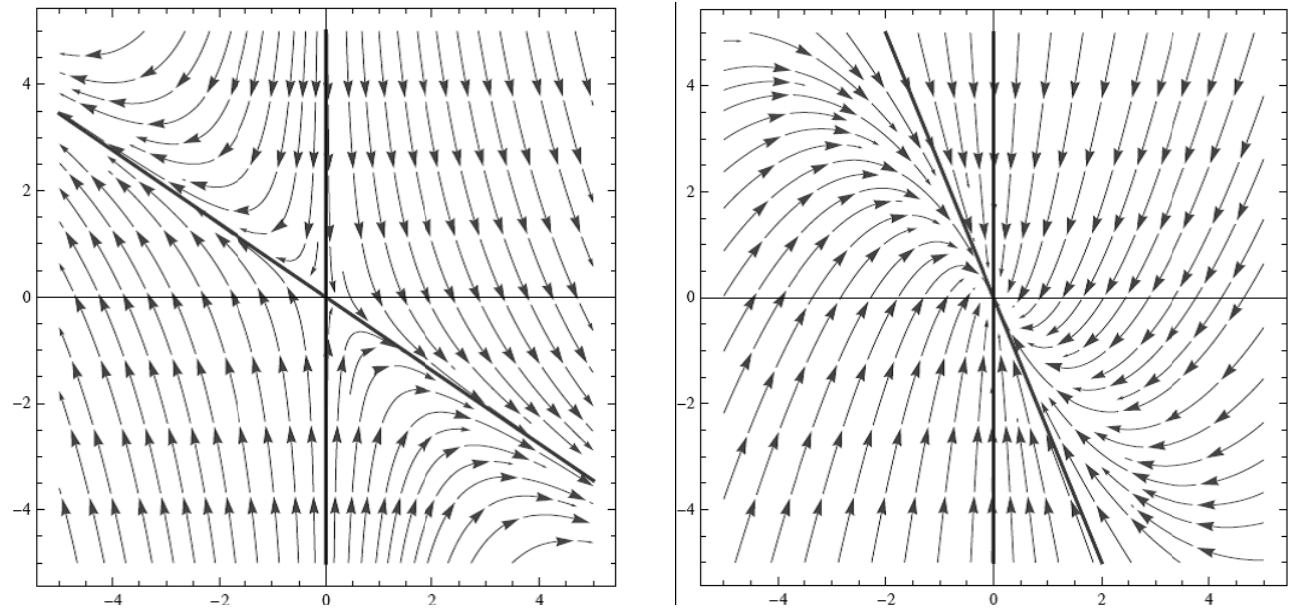

Figure 2. Eigensystem and phase portrait of the linear system (5) for $\beta=2$.

Equilibrium $\left(\boldsymbol{K}_{\mathbf{1}}, \mathbf{0}\right)$ : At the coordinates point of the equilibria, following expressions gives the linear system

$$
f_{u}\left(K_{1}, 0\right)=-r_{1} ; f_{w}\left(K_{1}, 0\right)=-r_{1} ; h_{u}\left(K_{1}, 0\right)=0 \text { and } h_{w}\left(K_{1}, 0\right)=\frac{r_{2}\left(K_{2}-K_{1}\right)}{\left(K_{2}+\beta K_{1}\right)}
$$

Now given nonlinear system converted to a linear system as

$$
\begin{aligned}
& \frac{d u}{d t}=-r_{1}\left(u+w-K_{1}\right) \\
& \frac{d w}{d t}=\frac{r_{2}\left(K_{2}-K_{1}\right)}{\left(K_{2}+\beta K_{1}\right)} w
\end{aligned}
$$

and the linearization matrix at $\left(K_{1}, 0\right)$ is 


$$
M_{\left(K_{1}, 0\right)}=\left(\begin{array}{cc}
-r_{1} & -r_{1} \\
0 & r_{2}\left(K_{2}-K_{1}\right) /\left(K_{2}+\beta K_{1}\right)
\end{array}\right)
$$

corresponding eigenvalues of $M_{\left(0, K_{2}\right)}$ are $\lambda_{1}=-r_{1}$ and $\lambda_{2}=R_{2}\left(K_{2}-K_{1}\right) /\left(K_{2}+\beta K_{1}\right)$, here $\lambda_{2}>0$ if $K_{2}>K_{1}$ and $\lambda_{2}<0$ for $K_{2}<K_{1}$. In particular, choosing the parameters $r_{1}=3, r_{2}=5$ and $\beta=3$, then for $K_{1}=3, K_{2}=4$, we have $\lambda_{1}=-3, \lambda_{2}=5 / 3$ with eigenvectors

$$
\left(\begin{array}{l}
0 \\
1
\end{array}\right) \text { and }\left(\begin{array}{c}
-29 / 44 \\
1
\end{array}\right) \text {, }
$$

respectively. The eigenvalues of the matrix $M_{\left(0, K_{2}\right)}$ are both real and have oppsite sign. So, we conclude that the equilibrium $\left(K_{1}, 0\right)$ is an unstable saddle point (see figure 3 , left). In the biological concept, it means the growth of the species $u$ is no longer sustainable in a given habitat during the competition.

Likewise, for $K_{1}=4, K_{2}=3$ with same values of the other parameters mentioned above, we have $\lambda_{1}=-3, \lambda_{2}=5 / 3$ with eigenvectors

$$
\left(\begin{array}{l}
0 \\
1
\end{array}\right) \text { and }\left(\begin{array}{c}
-29 / 34 \\
1
\end{array}\right) \text {, }
$$

respectively.

Since both eigenvalues are negative, we can summarize the critical point $\left(K_{1}, 0\right)$ is an asymptotically stable point shown in figure 3 (right). So, in this circumstance as the species $w$ will be extinct from the given ecological niche.
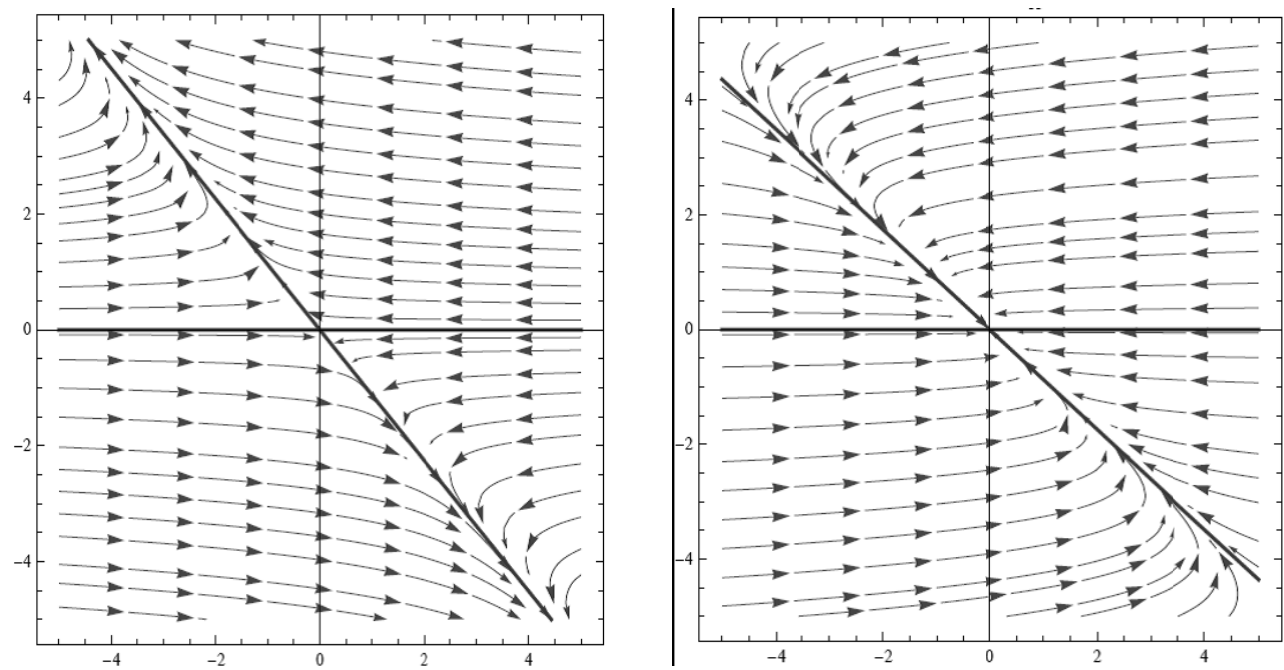

Figure 3. Eigensystem and phase portrait of the linear system (6). 


\section{Numerical Solutions}

For numerical tests, consider the Runge-Kutta methods of order four to solve the system of initial value problem (1).


Figure 4. Numerical solutions of the Logistic-Food limited growth system of equations (1) for $\beta=0.5, r_{1}=r_{2}$ $=1.0$ and (left) $K_{1}=3.0, K_{2}=2.0$, (right) $K_{1}=2.0=K_{2}$ with initial values $u_{0}=\mathrm{w}_{0}=2.0$.

For $r_{1}=r_{2}=1.0$ and different carrying capacities, $K_{1}>K_{2}$, the solution of the logistic equation coincides with the carrying capacity $K_{1}$, while the solution of the Food-limited equation tends to 0 for $\beta=0.5$ (see Fig. 4, left). When $K_{1}=K_{2}$, both population coexist as shown in Fig. 4 (right). Both populations are cooperating with each other when their resource distributions are equal even though the growth functions are different.

\section{Conclusion}

In sum, the considered model suggests that carrying capacity plays an important role in determination of the competition outcome between two species in a given ecological niche. If carrying is identical, there is a possible coexistence of both species. It is also notable that the parameter $\beta$ and intrinsic growth rates are important factors to determine which species will survive. This model can also be applicable for particular species in any habitats.

\section{Acknowledgment}

The authors are grateful to the reviewers for their constructive suggestions to enrich the manuscript. 


\section{REFERENCES}

[1] Gause, G. F. Struggle for Existence, Hafner, New York, 1934.

[2] Gilpin, M. E. and Ayala, F. J. Global models of growth and competition, Proc. Natl. Acad. Sci. USA, 1973, 3590-3593.

[3] Gompertz, B. On the nature of the function expressive of human mortality and on a new mode of determining the value of life contingencies, Philos. Trans. Roy. Soc. London, 1825, 513-583.

[4] Hale, J. K. Ordinary Differential Equations, John Wiley and Sons, New York, 1969.

[5] LaSalle, J. P. The stability of dynamical system, Regional Conference Series in Applied Mathematics. SIAM. Philadelphia, 1976.

[6] Perko, L. Differential Equations and Dynamical Systems, Springer, Third edition, 2008.

[7] Smith, F. E. Population dynamics in Daphnia Magna and a new model for population, Ecology 4(1963), 651-663.

[8] Strogatz, S. H. Nonlinear Dynamics and Chaos, With Applications to Physics, Biology, Chemistry, and Engineering. Westview Press, Cambridge, 2000.

[9] Verhulst, P.F. Notice sur la loi que la populations suit dans son accroissement, Correspondence Mathematique et Physique, 1838, 113-121.

[10] Wiggins, S. Introduction to Applied Nonlinear Dynamical Systems and Chaos, Springer-Verlag, New York, 1983. 